\title{
BIP NACH REGIONEN
}

Die Unterschiede zwischen der Wirtschaftsleistung verschiedener OECD-Länder sind oft geringer als zwischen einzelnen Regionen innerhalb dieser Länder. Im Zeitverlauf sind diese regionalen Disparitäten zudem bestehen geblieben, selbst wenn sich die Unterschiede zwischen den Ländern verringert haben.

\section{Definition}

Die regionalen Unterschiede in der Wirtschaftsleistung werden hier am regionalen Pro-Kopf-BIP oder BIP je Erwerbstätigen beurteilt. Das BIP eines Landes oder einer Region wird gemäß den Definitionen des Systems der Volkswirtschaftlichen Gesamtrechnungen von 1993 (SNA 1993) gemessen.

Der Gini-Index misst die Ungleichheit zwischen allen Regionen eines gegebenen Landes. Der Index nimmt Werte zwischen 0 und 1 an, wobei ein Wert von 0 bedeutet, dass keine Ungleichheit besteht. Er misst jeder Region ungeachtet ihrer Größe dasselbe Gewicht bei; daher sind Unterschiede bei den Indexwerten zwischen den Ländern z.T. auf Differenzen in der Durchschnittsgröße der Regionen in jedem Land zurückzuführen.

Während es bei der Analyse der Einkommensungleichheit naheliegt, auf der Personenebene anzusetzen, lässt sich bei der Untersuchung regionaler wirtschaftlicher Ungleichgewichte keine so offensichtliche Parallele ziehen. Die Größe von Regionen kann sowohl von Land zu Land als auch innerhalb einzel-

\section{Überblick}

Die Produktivitätsunterschiede zwischen einzelnen Regionen ein und desselben Landes sind oft beträchtlich. Große Unterschiede bestehen in Chile, der Türkei, dem Vereinigten Königreich und Frankreich.

In der Regel ist ein großer Teil des nationalen BIP-Wachstums einer kleinen Zahl von Regionen zuzuschreiben. Im Durchschnitt zeichneten im Zeitraum 1995-2009 knapp $10 \%$ der Regionen für $42 \%$ des Wachstums im OECD-Raum verantwortlich. Auf Länderebene war der regionale Wachstumsbeitrag in Ungarn, Griechenland, Schweden, Finnland, Japan, Spanien und dem Vereinigten Königreich sowie bei den Nicht-OECD-Ländern in der Russischen Föderation besonders konzentriert. In den genannten Ländern war über die Hälfte des nationalen Wachstums zwischen 1995 und 2009 den 10\% der Regionen mit dem höchsten BIP-Wachstum zuzuschreiben.

Der Gini-Index ist eine Messgröße der Ungleichheit, die jeder Region eines Landes unabhängig von ihrer Größe dasselbe Gewicht beimisst, während die Zahl der in Regionen mit niedrigem Pro-Kopf-BIP (unter dem nationalen Medianwert) lebenden Personen über die unterschiedlichen wirtschaftlichen Folgen von Disparitäten innerhalb eines Landes Auskunft gibt. Obwohl beispielsweise die anhand des Gini-Index gemessenen regionalen Disparitäten beim Pro-Kopf-BIP in der Slowakischen Republik, Chile, der Türkei und Estland dieselbe Größenordnung aufweisen, variiert der in Regionen mit niedrigem ProKopf-BIP lebende Anteil der Landesbevölkerung zwischen nahezu 54\% in der Slowakischen Republik und 23\% in Estland. ner Länder sehr unterschiedlich sein, so dass der Grad der geografischen Konzentration und territorialen Disparität schon allein von der Definition einer Region abhängt. Bei größeren Regionen gleichen sich territoriale Unterschiede in der Regel aus und die Disparitäten nehmen ab.

\section{Vergleichbarkeit}

Wie bei den anderen regionalen Statistiken wird die Vergleichbarkeit durch Unterschiede in der Bedeutung des Begriffs „Region“ beeinträchtigt. Der Begriff „Region“ kann sowohl innerhalb einzelner Länder als auch von Land zu Land ganz unterschiedliche Inhalte mit signifikanten Abweichungen im Hinblick auf Fläche und Bevölkerung haben. Die OECD hat daher eine Klassifizierung der Regionen in jedem der Mitgliedsländer vorgenommen. Die Klassifizierung unterscheidet zwei territoriale Ebenen: eine obere (TL 2, Makroregionen) und eine untere Ebene (TL 3, Mikroregionen). Alle hier angegebenen Daten beziehen sich auf Mikroregionen, mit Ausnahme von Australien, Brasilien, Chile, China, Indien, Kanada, Mexiko, der Russischen Föderation, Südafrika, der Türkei und den Vereinigten Staaten.

„2009 oder letztes verfügbares Jahr“ bezieht sich für alle Länder auf 2009, mit Ausnahme Chiles (2007), Norwegens (2007), Schwedens (2007) und der Türkei (2008). „1995-2009 oder letzter verfügbarer Zeitraum" bezieht sich für alle Länder auf Daten der Jahre 1995-2009, außer für Estland (1996-2009), Norwegen (1997-2007), Polen (1999-2009), die Türkei (2004-2008), China (2004-2008), Indien (2000-2008), die Russische Föderation (2005-2008) und Schweden (1995-2007).

\section{Quelle}

- OECD Regional Database.

- OECD (2011), OECD Regions at a Glance, OECD Publishing.

\section{Weitere Informationen}

\section{Analysen}

- OECD (2012), OECD Latin American Economic Outlook, OECD Publishing.

- OECD (2012), Promoting Growth in All Regions, OECD Publishing.

- OECD (2012), Southeast Asian Economic Outlook, OECD Publishing.

- OECD (2011), OECD Territorial Reviews, OECD Publishing.

- OECD (2011), Regional Outlook 2011, OECD Publishing.

- OECD (2009), How Regions Grow: Trends and Analysis, OECD Publishing.

- OECD (2009), Regions Matter: Economic Recovery, Innovation and Sustainable Growth, OECD Publishing.

\section{Online-Datenbanken}

- OECD Regional Database.

\section{Websites}

- Regional Statistics and Indicators, www.oecd.org/gov/regional/ statisticsindicators. 
Bandbreite des regionalen Pro-Kopf-BIP

In Prozent des nationalen Pro-Kopf-BIP, 2009 oder letztes verfügbares Jahr

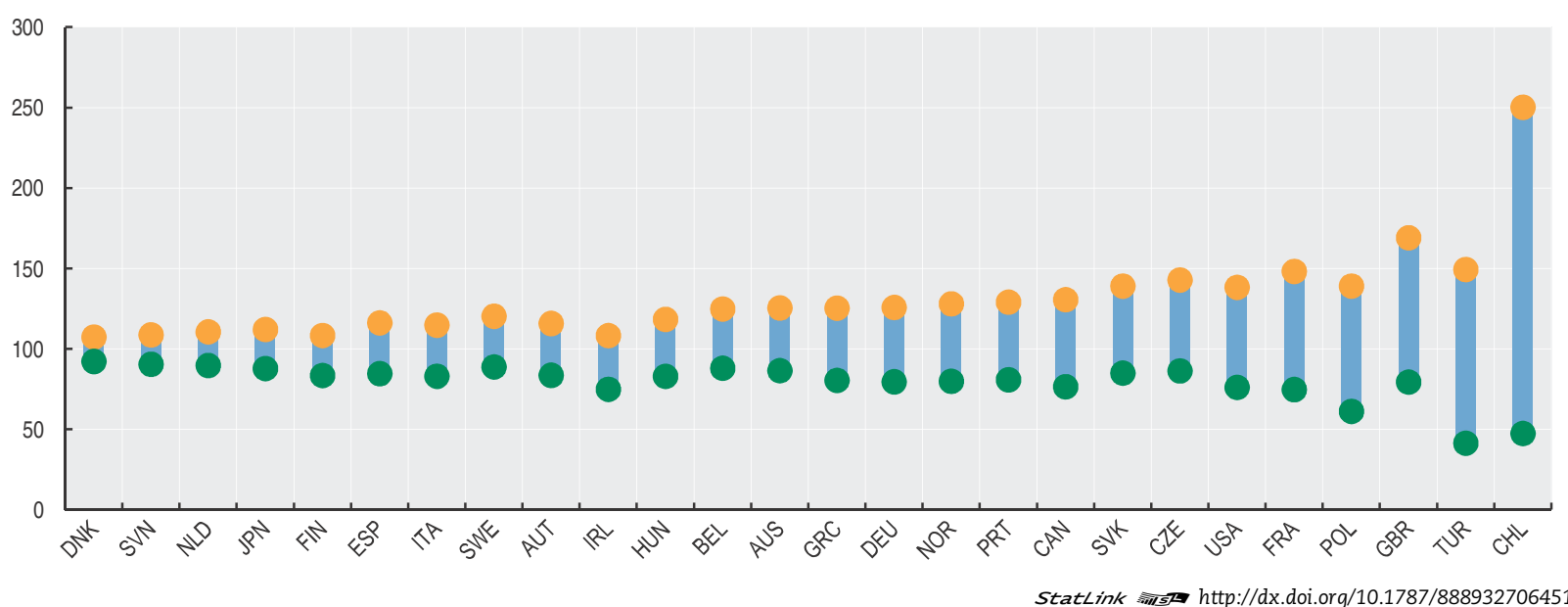

Anteil des BIP-Anstiegs, der auf die dynamischsten $10 \%$ der Regionen jedes Landes entfällt

In Prozent, 1995-2009 oder letzter verfügbarer Zeitraum

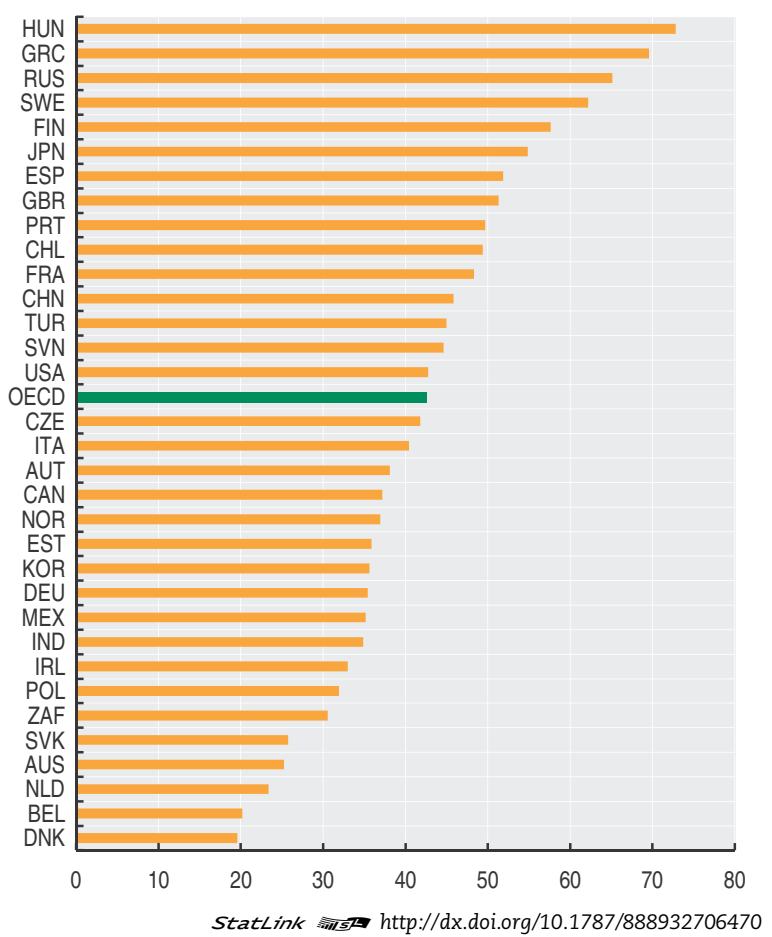

Gini-Index des regionalen Pro-Kopf-BIP und Anteil der Bevölkerung in Regionen mit geringem Pro-Kopf-BIP

2009 oder letztes verfügbares Jahr

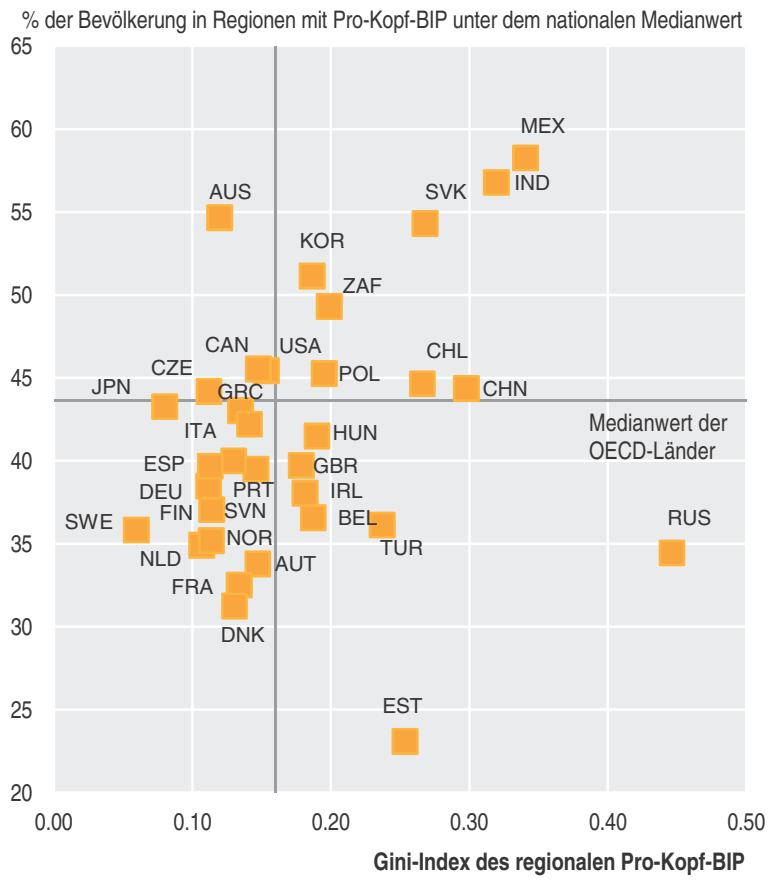

StatLink तiोs http://dx.doi.org/10.1787/888932706489 


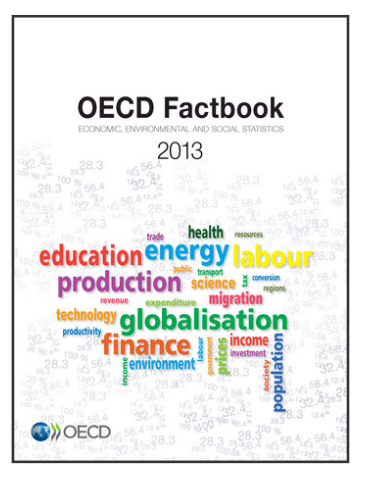

\section{From: \\ OECD Factbook 2013 \\ Economic, Environmental and Social Statistics}

Access the complete publication at:

https://doi.org/10.1787/factbook-2013-en

Please cite this chapter as:

OECD (2013), "BIP nach Regionen", in OECD Factbook 2013: Economic, Environmental and Social Statistics, OECD Publishing, Paris.

DOI: https://doi.org/10.1787/factbook-2013-12-de

Das vorliegende Dokument wird unter der Verantwortung des Generalsekretärs der OECD veröffentlicht. Die darin zum Ausdruck gebrachten Meinungen und Argumente spiegeln nicht zwangsläufig die offizielle Einstellung der OECDMitgliedstaaten wider.

This document and any map included herein are without prejudice to the status of or sovereignty over any territory, to the delimitation of international frontiers and boundaries and to the name of any territory, city or area.

You can copy, download or print OECD content for your own use, and you can include excerpts from OECD publications, databases and multimedia products in your own documents, presentations, blogs, websites and teaching materials, provided that suitable acknowledgment of OECD as source and copyright owner is given. All requests for public or commercial use and translation rights should be submitted to rights@oecd.org. Requests for permission to photocopy portions of this material for public or commercial use shall be addressed directly to the Copyright Clearance Center (CCC) at info@copyright.com or the Centre français d'exploitation du droit de copie (CFC) at contact@cfcopies.com. 